\title{
EDITORIAL
}

\section{HUMAN MICROBIOTA}

\author{
BY \\ Basma Kamal Ramadan \\ Physiology Department, \\ Faculty of Medicine (Girls), Al-Azhar University, Cairo
}

Microbes are usually live in communities, and each of these communities has a distinct taxonomical structure. Due to its close relationship with human health and disease, the human microbiome has received great attention and is probably considered to be the most valuable biomarker in preventing and solving human diseases (Cong and Zhang, 2018).

Microbiota is an "ecological community of commensal, symbiotic and pathogenic organisms". It is found in and on all multicellular organisms studied to date from plants to animals. The human microbiota is the aggregate of microorganisms on or within any of a number of human tissues and biofluids, including the skin, mammary glands, placenta, seminal fluid, uterus, ovarian follicles, lung, saliva, oral mucosa, conjunctiva, biliary and gastrointestinal tracts. The human microbiome refers specifically to the collective genomes of resident microorganism (Sherwood et al., 2013).

They include bacteria, archaea, fungi, protists and viruses. Bacterial type is present mainly in human mouth, vagina and GIT (Sommer and B?ckhed, 2013). Archaea are present in the human gut, but, in contrast to the enormous variety of bacteria in this organ, the numbers of archaeal species are much more limited (Eckburg et al., 2005). Fungi in particular yeasts, are present in the human gut. The best-studied of these are Candida species due to their ability to become pathogenic in immuno-compromised and even in healthy hosts. Yeasts are also present on the skin, such as Malassezia species, where they consume oils secreted from the sebaceous glands (Erdogan and Rao, 2015). Viruses especially bacterial viruses (bacteriophages), colonize various body sites. These colonized sites include the skin, gut, lungs, and oral cavity. Virus communities have been associated with some diseases, and do not simply reflect the bacterial communities (Abeles et al., 2014).

\section{Relative number of microbiota:}

In 2014, the American Academy of Microbiology published a FAQ that emphasized that the number of microbial cells and the number of human cells are both estimates, and noted that the human microbiome includes around 100 trillion bacterial cells and that an adult human typically has around 10 trillion human cells.

\section{Gut Flora:}

The gut flora has the largest numbers of bacteria and the greatest number of species compared to other areas of the body (Quigley, 2013). In humans, the composition of gut 
flora is established during birth. Birth by Cesarean section or vaginal delivery also influences the gut's microbial composition. Babies born through the vaginal canal have non-pathogenic, beneficial gut microbiota similar to those found in the mother (Mueller et al., 2015). However, the gut microbiota of babies delivered by cesarean section harbors more pathogenic bacteria such as Escherichia coli and Staphylococcus, and it takes longer to develop non-pathogenic beneficial gut microbiota (Wall et al., 2009).

Bacteria can be transferred from mother to child through direct contact and after birth. The infant microbiome is established, commensal bacteria quickly populate the gut, prompting a range of immune responses, and "programming" the immune system with long-lasting effects (Cahenzli et al., 2012).

\section{Skin Flora:}

The human skin microbiota play an important role in the generation of human odors (Verhulst et al., 2010 and Bouslimani et al., 2015). Human odor profiles include more than 350 identified compounds (Bernier et al., 2000), and it has been shown that bacteria on human skin are involved in the release of approximately 150 volatile organic compounds (hereafter referred to as VOCs) (Verhulst et al., 2009). Some of these VOCs released by bacteria are typically found in human odor (Bouslimani et al., 2015).

\section{Importance of gut microbiota:}

Some human gut microorganisms benefit the host by fermentating dietary fiber into short-chain fatty acids (SCFAs), such as acetic acid and butyric acid, which are then absorbed by the host. Intestinal bacteria also play a role in synthesizing vitamin $\mathrm{B}$ and vitamin $\mathrm{K}$ as well as metabolizing bile acids, sterols, and xenobiotics (Sherwood et al., 2013).

Changes in the composition of gut microbiota has also been found to be correlated with harmful effects on health, from these effects is cancer. Microorganisms are implicated in about $20 \%$ of human cancers. Particularly for potential factors in colon cancer, bacterial density is one million times higher than in the small intestine. Approximately, 12-fold more cancers occur in the colon compared to the small intestine, possibly establishing a pathogenic role for microbiota in colon and rectal cancers. Microbial density may be used as a prognostic tool in assessment of colorectal cancers (Gagnière et al., 2016). The microbiota may affect carcinogenesis in three broad ways: (i) altering the balance of tumor cell proliferation and death, (ii) regulating immune system function, and (iii) influencing metabolism of host-produced factors, foods and pharmaceuticals. International Agency for Research on Cancer stated that Microbes may secrete proteins or other factors directly drive cell proliferation in the host, or may up- or down-regulate the host immune system including driving acute or chronic inflammation in ways that contribute to carcinogenesis (Garrett, 2015).

\section{REFERENCES}

- $\quad$ Abeles, S.R.; Robles-Sikisaka, R.; Melissa, L.; Andrew, G.; Salzman, J.; Boehm, T.K. and Pride, D.T. (2014): Human oral viruses are personal, persistent and gender-consistent. The ISME Journal, 8(9): 1753-1767.

- American Academy of Microbiology FAQ (2014): Human Microbiome January 2014

- $\quad$ Bernier, U.R.; Kline, D.L.; Barnard, D.R.; Schreck, C.E. and Yost, R.A. (2000): Analysis of human skin emanations by gas chromatography/mass spectrometry. Anal Chem.; 72: 747-756. 
- Bouslimani, A.; Porto, C.; Rath, C.M.; Wang, M.; Guo, Y. and Gonzalez, A. (2015): Molecular cartography of the human skin surface in 3D. Proc Natl Acad Sci USA.; 112: E2120-E2129.

- Cahenzli, J.; Balmer, M. L. and McCoy, K. D. (2012): Microbial-immune cross-talk and regulation of the immune system. Immunology. 138 (1): 12-22.

- Cong J and Zhang X. (2018): How human microbiome talks to health and disease. Eur J Clin Microbiol Infect Dis.(In Press).

- Eckburg, P.B.; Bik, E.M. and Bernstein, C.N. (2005): Diversity of the human intestinal microbial flora. Science, 308(5728): 1635-8.

- Erdogan, A. and Rao, S.S. (2015): Small intestinal fungal overgrowth. Curr Gastroenterol Rep., 17 (4): 16.

- $\quad$ Gagnière, J.; Raisch, J.; Veziant, J.; Barnich, N.; Bonnet, R.; Buc, E.; Bringer, M. A.; Pezet, D. and Bonnet, M. (2016): Gut microbiota imbalance and colorectal cancer. World Journal of Gastroenterology, 22 (2): 501-518.

- Garrett, W.S. (2015): Cancer and the microbiota. Science. 348 (6230): 80-6.

- Mueller, N.T.; Bakacs, E.; Combellick, J.; Grigoryan, Z. and Dominguez-Bello, M.G. (2015): The infant microbiome development: mom matters. Trends in Molecular Medicine, 21 (2): 109-117.

- Quigley, E.M. (2013): Gut bacteria in health and disease. Gastroenterol Hepatol (N Y), 9 (9): 560-9.

- Sherwood, L.; Willey, J. and Woolverton, C. (2013): Prescott's Microbiology (9th ed.). Pbl. New York: McGraw Hill, pp. 713-721.

- Sommer, F. and B?ckhed, F. (2013): The gut microbiota-masters of host development and physiology. Nat Rev Microbiol.; 11 (4): 227-38.

- Verhulst, N.O.; Beijleveld, H.; Knols, B.G.J.; Takken, W.; Schraa, G. and Bouwmeester, H.J. (2009): Cultured skin microbiota attracts malaria mosquitoes. Malar J., pmid:20017925

- Verhulst, N.O.; Takken, W.; Dicke, M.; Schraa, G. and Smallegange, R.C. (2010): Chemical ecology of interactions between human skin microbiota and mosquitoes. FEMS Microbiol Ecol.; 74: 19.

- Wall, R.; Ross, R.P.; Ryan, C.A.; Hussey, S.; Murphy, B.; Fitzgerald, G.F and Stanton, C. (2009): Role of Gut Microbiota in Early Infant Development. Clinical Medicine, Pediatrics, 3: 45-54.

\section{Basma Kamal Ramadan \\ Physiology Department, Faculty of Medicine (Girls), Al-Azhar University, Cairo}

